\title{
Methanol Maser Parallaxes and Proper Motions
}

\author{
Y. Xu' ${ }^{1}$, M. J. Reid ${ }^{2}$, L. Moscadelli ${ }^{3}$, K. M. Menten ${ }^{4}$, X. W. Zheng ${ }^{5}$, \\ A. Brunthaler ${ }^{4}$, B. Zhang ${ }^{4}$, K. L. J. $\operatorname{Rygl}^{6}$, J. J. $\mathbf{L i}^{1}$, and A. Sanna ${ }^{4}$ \\ ${ }^{1}$ Purple Mountain Observatory, China \\ email: xuye@pmo.ac.cn \\ ${ }^{2}$ Harvard-Smithsonian Center for Astrophysics, USA \\ ${ }^{3}$ Arcetri Obs., Italy \\ ${ }^{4}$ Max-Planck-Institute für Radioastronomie, Germany \\ ${ }^{5}$ Nanjing University, China \\ ${ }^{6}$ INAF-IAPS, Italy
}

\begin{abstract}
Due to their compactness, persistence and slow motion, Class II $\mathrm{CH}_{3} \mathrm{OH}$ masers are excellent targets for parallax and proper motion measurements for massive star-forming regions in the Galactic Disk. These measurements can be used to improve our understanding of the spiral structure and dynamics of the Milky Way. At the same time, Class $\mathrm{II} \mathrm{CH}_{3} \mathrm{OH}$ masers can also be used to study gas kinematics close to the exciting star, tracing rotation, infall and/or outflow motions.
\end{abstract}

Keywords. $\mathrm{CH}_{3} \mathrm{OH}$ maser, parallaxes and proper motions, Galactic structure

\section{Introduction}

Class II $\mathrm{CH}_{3} \mathrm{OH}$ masers at 6.7 and $12.2 \mathrm{GHz}$ are strong (10-100 Jy), compact (typical spot size of a few milli-arcseconds (mas)), persistent (over many years), and are generally associated with slowly moving gas (velocities $\leqslant 10 \mathrm{~km} \mathrm{~s}^{-1}$ ). Thus, they are excellent astrometric targets. About a thousand $6.7 \mathrm{GHz}$ and a hundred $12.2 \mathrm{GHz} \mathrm{CH}_{3} \mathrm{OH}$ masers have been detected. Large numbers are located in the "molecular ring" and share the same spatial distribution as CO gas. Since the 6.7 and $12.2 \mathrm{GHz}$ masers are always found in regions of on-going massive star-formation, they are good tracers of the spiral structure in the Galactic disk. In addition, they can also be used to infer the three-dimensional (3-D) gas kinematics around the massive (proto)stars at radii of tens to hundreds of astronomical unit (AU), which is not accessible via interferometric observations of thermal tracers.

\section{Source of astrometric error}

The spiral structure of the Milky Way is still poorly known. The classical model is mainly based on the kinematic distances (Georgelin \& Georgelin 1976), which have been shown to be inaccurate ( $\mathrm{Xu}$ et al. 2006) by up to $30 \%$ or more. Masers allow us a direct measurement of distances via trigonometric parallaxes; however, a (relative) positional accuracy of about $10 \mu$ as is necessary to determine accurate distances throughout the Milky Way. Achieving such an accuracy with Very Long Baseline Interferometry (VLBI) observations at centimeter wavelengths is not an easy task. Many factors can degrade the measurement accuracy. Systematic errors are related to the separation angle between target and calibrator, motivating one to find background calibrators close to the target. 
Either the (maser) target or the background sources can be used as a phase-reference calibrator. If the maser target is strong enough to be detected within the interferometer coherence time, a quasar (QSO) as weak as a few $\mathrm{mJy} /$ beam can be revealed by imaging after applying the phase corrections derived from the maser. Statistically, the weaker the QSO, the higher the chance for it to be found near the maser. Using several interferometers (VLA, ATCA, MERLIN and VLBA), we have searched for weak QSOs and determined their positions accurately. Typically we find 3 suitable QSOs within 1 to 2 degrees of any $\mathrm{CH}_{3} \mathrm{OH}$ maser source. Using multiple background calibrators helps in reducing systematic errors due to time variation in the atmosphere and in calibrator structure.

Poor knowledge of the vertical atmospheric delay for each station of the VLBI array is often the major source of systematic error in position. At high elevation angles, the VLBA correlator model is typically in error by about $10 \mathrm{~cm}$ of excess path length at $12 \mathrm{GHz}$, while at $6.7 \mathrm{GHz}$ the model inaccuracy can be larger than $20 \mathrm{~cm}$, dominated by ionospheric path delay. To achieve an astrometric accuracy $\leqslant 50 \mu$ as, it is crucial to remove the residual tropospheric and ionospheric effects, in particular for low-frequency observations. One way to measure and remove the tropospheric delays involves geodeticlike observing blocks. By observing about 15 QSOs, whose positions are known to better than 1 mas, the residual atmospheric delays above each antenna can be estimated accurately. Ionospheric (dispersive) delays can be partially removed by applying a global ionospheric model derived from GPS measurements. Our VLBA observations always include geodetic-like observing blocks and correct for the GPS ionospheric model during the data calibration.

One other source of observational error comes from the absolute position of the phasereference calibrator. If the error in the correlated position of the calibrator is $\geqslant 10$ mas, the phase-referenced image can be distorted and affected by second-order positional errors. To be able to detect weak (a few mJy/beam) signals in the phase-reference map, it is therefore important to know the calibrator position with high accuracy. Note that most of the VLBA calibrators have a position accuracy of $\sim 1$ mas.

\section{Parallaxes and proper motions}

After calibrating and removing the sources of error discussed in the previous section, one can measure parallaxes and proper motions of the $\mathrm{CH}_{3} \mathrm{OH}$ masers with high accuracy. Figure 1 shows the results for the $12 \mathrm{GHz}$ masers towards the UC HII region W3(OH). It displays the sky-projected trajectory of a single $12 \mathrm{GHz}^{\mathrm{CH}} \mathrm{CH}_{3} \mathrm{OH}$ maser spot relative to three QSOs, which results from the sum of the annual parallax, Solar Motion, differential Galactic rotation, peculiar motion (of the target star with respect to its Local standard of rest (LSR)) and internal motion (of the maser spot with respect to the star. The projection of the maser spot path on each equatorial coordinate (RA and Dec) can be fitted with a linear plus a sinusoidal motion, the latter reflecting the annual parallax. For $\mathrm{W} 3(\mathrm{OH})$, we obtain a parallax of $512 \pm 10 \mu$ as and spot proper motions are derived with an accuracy of $\sim 1 \mathrm{~km} \mathrm{~s}^{-1}$ (Xu et al. 2006). Such a parallax accuracy is enough to measure distances to $\sim 10 \mathrm{kpc}$, comparable with the size of the Milky Way disk, with $10 \%$ uncertainty.

In the last years, the $6.7 \mathrm{GHz}$ methanol masers were successfully used for parallax measurements with the European VLBI Network, achieving accuracies approaching 20 $\mu$ as (Rygl et al. 2010). The obtained distances were found to be in agreement with VERA water masers parallaxes (Sato et al. 2008, Nagayama et al. 2011) for two starforming regions. Recently, the distance to the Cygnus $\mathrm{X}$ region was measured using 6.7 
$\mathrm{GHz}$ methanol masers. The parallaxes toward four star-forming regions, W 75N, DR 21, DR 20, and IRAS 20290+4052 in Cygnus X North, were found to be consistent with a single distance of $1.40 \pm 0.08 \mathrm{kpc}$ for the Cygnus X complex (Rygl et al. 2012). Figure 2 shows the parallax fit of $\mathrm{W} 75 \mathrm{~N}$ based on 10 masers spots observed in eight EVN epochs. The resulting parallax was $0.772 \pm 0.042$ mas, which corresponds to a distance of $1.30 \pm 0.07 \mathrm{kpc}$.

To date, the $\mathrm{CH}_{3} \mathrm{OH}$ masers have been used to determine, parallaxes and proper motions for 26 massive star-forming regions (Bartkiewicz et al. 2008, Reid et al. 2009a, Moscadelli et al. 2009, Xu et al. 2009, Xu et al. 2011, Zhang et al. 2009, Brunthaler et al. 2009, Sanna et al. 2009, Rygl et al. 2010, Rygl et al. 2012), tabulated in Table 1. Most of them have parallax accuracy of better than 10\%. Reid et al. (2009b) modeled 18 parallaxes available in 2009 and found that massive star-forming regions lag circular rotation by about $15 \mathrm{~km} \mathrm{~s}^{-1}$. This provided motivation for a re-analysis of the Solar Motion by Schönrich et al. (2010), resulting in an increase in the component in the direction of Galactic rotation by $7 \mathrm{~km} \mathrm{~s}^{-1}$, and a corresponding reduction in the star forming region velocity lag.

We can update these model results based on the 26 parallax/proper motions. In the frame rotating with the Galaxy, taking the distance to the Galactic center of $8.3 \mathrm{kpc}$ and circular rotation speed of $239 \mathrm{~km} \mathrm{~s}^{-1}$ Brunthaler et al. (2011), and the updated Solar Motion values from Schönrich et al. (2010), we find an average lag of $12 \mathrm{~km} \mathrm{~s}^{-1}$ in the direction of Galactic rotation and an average motion of $11 \mathrm{~km} \mathrm{~s}^{-1}$ toward Galactic cen-
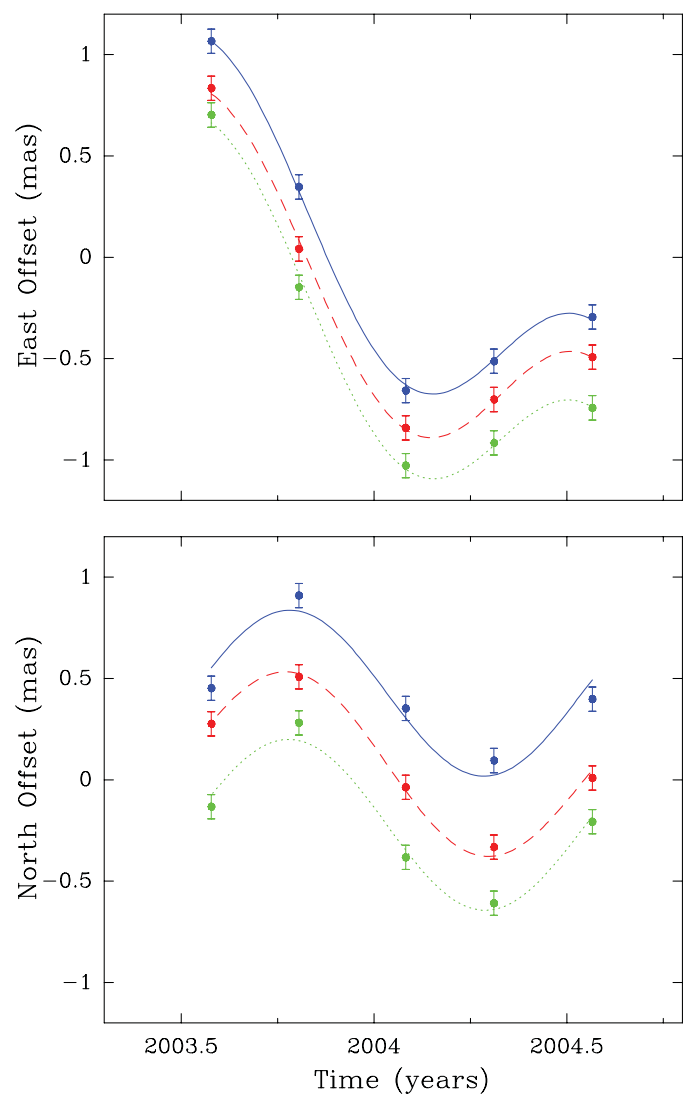

Figure 1. Position versus time for a maser spot relative to each QSO in W3OH. 
ter (see Figure 3) However, one can see that G9.62+0.20 \& G23.6-0.1 have large peculiar motions, probably caused by the gravitational potential of the bar making them unsuitable for studying average systematic motions. If we drop them and use the remaining 24 sources we find, similar to Reid et al. (2009b), on average a systematic motion of 5 $\mathrm{km} \mathrm{s}^{-1}$ toward the Galactic center and $7 \mathrm{~km} \mathrm{~s}^{-1}$ of counter rotation. Since the $\mathrm{CH}_{3} \mathrm{OH}$ masers are only associated with massive star-forming regions, this indicates that newly formed massive stars orbit the Milky Way $\sim 7 \mathrm{~km} \mathrm{~s}^{-1}$ slower than for circular orbits and $\sim 5 \mathrm{~km} \mathrm{~s}^{-1}$ towards the Galactic center. The systematic motions might be caused by a wrong estimate of the Solar Motion and an inadequate Galaxy rotation curve, while the accurate values would be obtained by the BeSSeL project.

\section{Gas kinematics close to the massive (proto)star}

Presently massive star-formation is a very active field of research but several theoretical issues associated with the ionization action and the dynamical effects of the radiation from the massive (proto)star on the surrounding gas are still unclear. However, the observation of massive star-forming regions is severely limited by the large distances (typically of several kpc) and the clustered formation mode, which prevent detailed studies of single (proto)stellar-systems, with all but VLBI observations of their associated maser emission.

For several years, we have been conducting a campaign of multi-epoch VLBI observations of the strongest interstellar molecular masers $\left(\mathrm{OH}\right.$ at $1.6 \mathrm{GHz}, \mathrm{CH}_{3} \mathrm{OH}$ at $6.7 \mathrm{GHz}$, and $\mathrm{H}_{2} \mathrm{O}$ at $22 \mathrm{GHz}$ ) towards a small sample of massive star-forming regions (Moscadelli et al. 2007, 2011; Sanna et al. 2010; Goddi et al. 2011). One of the major results of our observations is the result that the (VLBI measurable) 3-D velocity distribution of
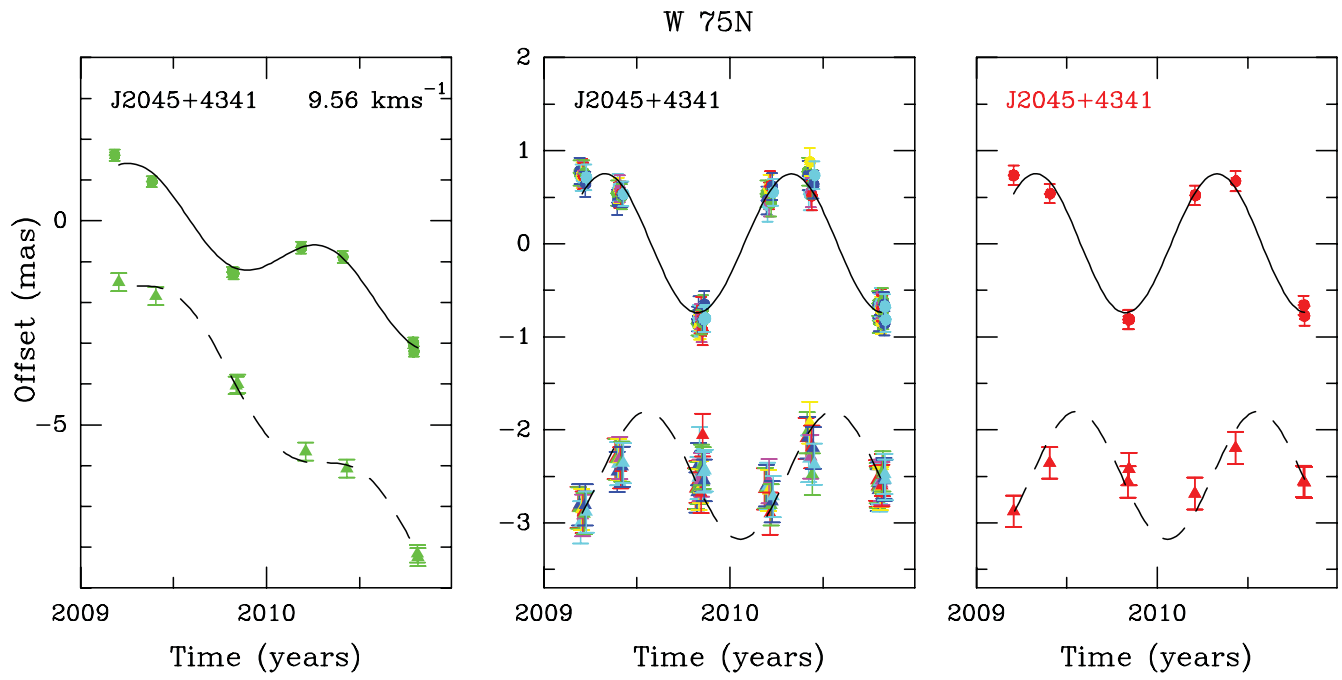

Figure 2. Results of the parallax fit for W $75 \mathrm{~N}$ using QSO J2045+4341 as position reference. Left panel: Results of the parallax fit for the maser spot at $V_{\mathrm{LSR}}=9.56 \mathrm{~km} \mathrm{~s}^{-1}$ without the removal of the proper motions. The right ascension and declination data have been offset for clarity. Middle and right panel: Results of the parallax fit for $\mathrm{W} 75 \mathrm{~N}$ after removing proper motions and positional offsets; combined fit on 10 maser spots (middle panel) and fit to the averaged data of the 10 maser spots (right panel). The declination data have been offset for clarity. The dots mark the data points in right ascension, while the filled triangles mark the declination data points. The solid lines show the resulting parallax fit in right ascension and the dashed lines show the fit in declination. The scale of the Y-axis of the middle and right panels is different from the scale of the left panel. 
Table 1. Parallaxes and Proper Motions of High-mass Star-Forming Regions.

\begin{tabular}{|c|c|c|c|c|c|c|c|}
\hline Source & $\begin{array}{c}\mathrm{l} \\
(\mathrm{deg})\end{array}$ & $\begin{array}{c}\mathrm{b} \\
(\mathrm{deg})\end{array}$ & $\begin{array}{l}\text { Parallax } \\
\text { (mas) }\end{array}$ & $\begin{array}{c}\mu_{x} \\
\left(\operatorname{mas~yr}^{-1}\right)\end{array}$ & $\begin{array}{c}\mu_{y} \\
\left(\text { mas yr }^{-1}\right)\end{array}$ & $\begin{array}{c}v_{L S R} S^{-1} \\
\left(\mathrm{~km} \mathrm{~s}^{-1}\right)\end{array}$ & $\begin{array}{l}\text { Maser } \\
(\mathrm{GHz})\end{array}$ \\
\hline G99.62+0.20 & 9.62 & +0.20 & $0.194 \pm 0.023$ & $-0.580 \pm 0.054$ & $-2.49 \pm 0.27$ & $+2 \pm 5$ & 12 \\
\hline $\mathrm{G} 12.89+0.49$ & 12.89 & +0.49 & $0.452 \pm 0.029$ & $+0.20 \pm 0.04$ & $-1.74 \pm 0.76$ & $+34 \pm 3$ & 12 \\
\hline G15.03-0.68 & 15.03 & -0.68 & $0.505 \pm 0.033$ & $+0.68 \pm 0.05$ & $-1.42 \pm 0.09$ & $+20 \pm 3$ & 12 \\
\hline G $23.0-0.4$ & 23.01 & -0.41 & $0.218 \pm 0.017$ & $-1.72 \pm 0.04$ & $-4.12 \pm 0.30$ & $+81 \pm 3$ & 12 \\
\hline G $23.4-0.2$ & 23.44 & -0.18 & $0.170 \pm 0.032$ & $-1.93 \pm 0.10$ & $-4.11 \pm 0.07$ & $+97 \pm 3$ & 12 \\
\hline G $23.6-0.1$ & 23.66 & -0.13 & $0.313 \pm 0.039$ & $-1.32 \pm 0.02$ & $-2.96 \pm 0.03$ & $+83 \pm 3$ & 12 \\
\hline G27.36-0.16 & 27.36 & -0.16 & $0.125 \pm 0.042$ & $-1.81 \pm 0.08$ & $-4.11 \pm 0.26$ & $+92 \pm 3$ & 12 \\
\hline G $35.2-0.7$ & 35.20 & -0.74 & $0.456 \pm 0.045$ & $-0.18 \pm 0.06$ & $-3.63 \pm 0.11$ & $+28 \pm 3$ & 12 \\
\hline G $35.2-1.7$ & 35.20 & -1.74 & $0.306 \pm 0.045$ & $-0.71 \pm 0.05$ & $-3.61 \pm 0.17$ & $+42 \pm 3$ & 12 \\
\hline W 51 IRS 2 & 49.49 & -0.37 & $0.195 \pm 0.071$ & $-2.49 \pm 0.08$ & $-5.51 \pm 0.11$ & $+56 \pm 3$ & 12 \\
\hline G $59.7+0.1$ & 59.78 & +0.06 & $0.463 \pm 0.020$ & $-1.65 \pm 0.03$ & $-5.12 \pm 0.08$ & $+27 \pm 3$ & 12 \\
\hline ON 1 & 69.54 & -0.98 & $0.389 \pm 0.045$ & $-3.24 \pm 0.89$ & $-5.42 \pm 0.46$ & $+12 \pm 1$ & 6.7 \\
\hline IRAS $20126+4104$ & 78.12 & +3.63 & $0.61 \pm 0.02$ & $-4.14 \pm 0.13$ & $-4.14 \pm 0.13$ & $-3.5 \pm 3$ & 6.7 \\
\hline IRAS $20290+4052$ & 79.74 & +0.99 & $0.737 \pm 0.062$ & $-2.84 \pm 0.09$ & $-4.14 \pm 0.54$ & $-1.4 \pm 3$ & 6.7 \\
\hline DR 20 & 80.86 & +0.38 & $0.687 \pm 0.038$ & $-3.29 \pm 0.13$ & $-4.83 \pm 0.26$ & $-3.0 \pm 3$ & 6.7 \\
\hline DR 21 & 81.74 & +0.59 & $0.666 \pm 0.035$ & $-2.84 \pm 0.15$ & $-3.80 \pm 0.22$ & $-3.0 \pm 3$ & 6.7 \\
\hline W $75 \mathrm{~N}$ & 81.87 & +0.78 & $0.772 \pm 0.042$ & $-1.97 \pm 0.10$ & $-4.16 \pm 0.15$ & $+9.0 \pm 3$ & 6.7 \\
\hline L 1206 & 108.18 & +5.52 & $1.289 \pm 0.153$ & $+0.27 \pm 0.23$ & $-1.40 \pm 1.95$ & $-10 \pm 3$ & 6.7 \\
\hline Cep A & 109.87 & +2.11 & $1.430 \pm 0.080$ & $+0.50 \pm 1.10$ & $-3.70 \pm 0.20$ & $-10 \pm 5$ & 12 \\
\hline NGC 7538 & 111.54 & +0.78 & $0.378 \pm 0.017$ & $-2.45 \pm 0.03$ & $-2.44 \pm 0.06$ & $-57 \pm 3$ & 12 \\
\hline L 1287 & 121.30 & +0.66 & $1.077 \pm 0.039$ & $-0.86 \pm 0.11$ & $-2.29 \pm 0.56$ & $-18 \pm 3$ & 6.7 \\
\hline NGC 281-W & 123.07 & -6.31 & $0.421 \pm 0.022$ & $-2.69 \pm 0.16$ & $-1.77 \pm 0.11$ & $-32 \pm 3$ & 6.7 \\
\hline W $3(\mathrm{OH})$ & 133.95 & +1.06 & $0.512 \pm 0.010$ & $-1.20 \pm 0.20$ & $-0.15 \pm 0.20$ & $-45 \pm 3$ & 12 \\
\hline S 252 & 188.95 & +0.89 & $0.476 \pm 0.006$ & $+0.02 \pm 0.01$ & $-2.02 \pm 0.04$ & $+11 \pm 3$ & 12 \\
\hline S 255 & 192.60 & -0.05 & $0.628 \pm 0.027$ & $-0.14 \pm 0.54$ & $-0.84 \pm 1.76$ & $+8 \pm 3$ & 6.7 \\
\hline G $232.6+1.0$ & 232.62 & +1.00 & $0.596 \pm 0.035$ & $-2.17 \pm 0.06$ & $+2.09 \pm 0.46$ & $+23 \pm 3$ & 12 \\
\hline
\end{tabular}

water masers, emerging from shocked molecular gas at the interface between the fast flowing and the ambient material, efficiently trace the structure of (proto)stellar outflows close to (within 10-100 AU) the (proto)star. Our study also provides evidence that the methanol masers are found at (relatively) large separations from the protostellar jet

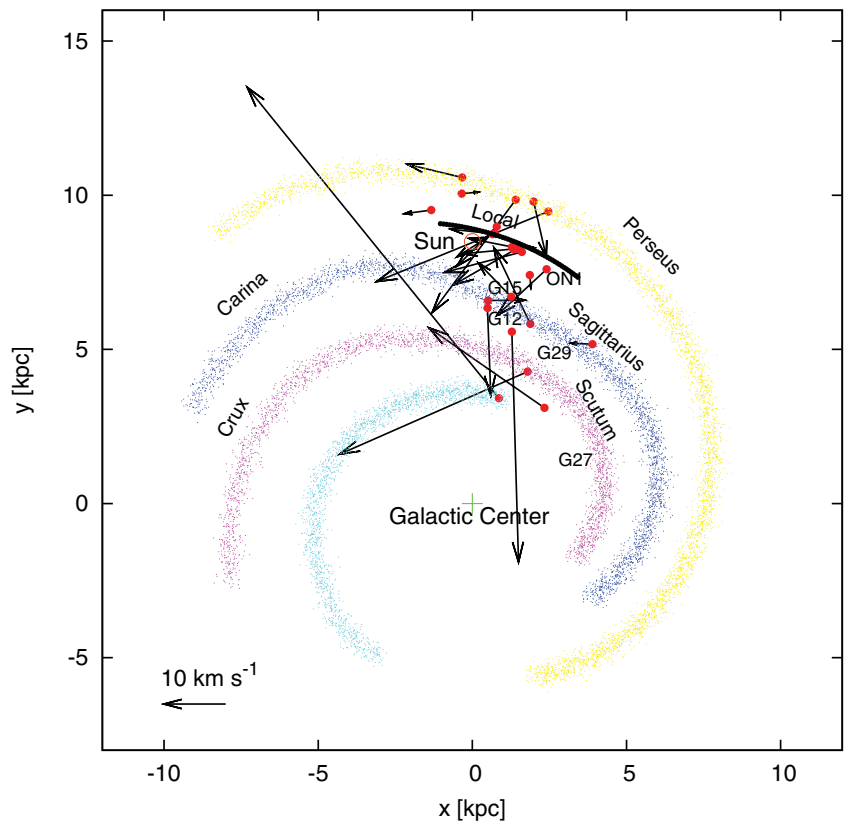

Figure 3. Peculiar motions of massive star forming regions. The amplitude scale is given at the bottom. 


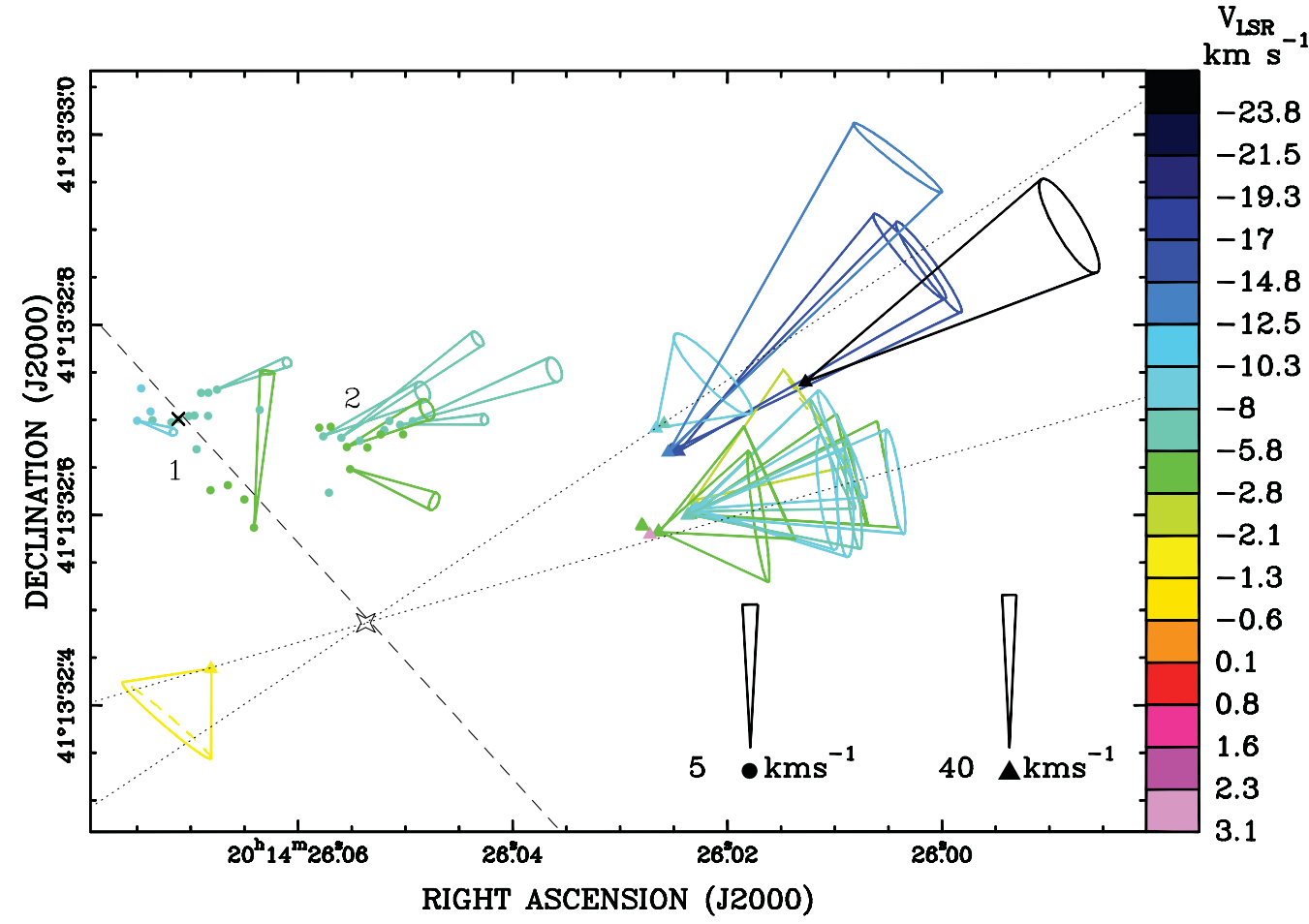

Figure 4. Water (triangles) and $6.7 \mathrm{GHz}$ methanol (circles) masers in IRAS 20126+4104. The cones indicate the $3 \mathrm{D}$ velocities of the maser features with respect to the star, for the $\mathrm{H}_{2} \mathrm{O}$ masers, or relative to a chosen feature (marked with a cross), for the $\mathrm{CH}_{3} \mathrm{OH}$ masers. Points without an associated cone have been detected only at one or two epochs and the associated proper motion cannot be computed or is considered unreliable. The cone opening angle corresponds to $1 \sigma$ motion uncertainties. The length of the cones is proportional to the speed, with different scales used for the two maser types as indicated in the figure. The colours correspond to the line of sight velocities, as shown in the colour scale to the right. The starred polygon marks the location of the star, obtained from the model fit to the $\mathrm{H}_{2} \mathrm{O}$ maser positions and 3D velocities. Labels "1" and "2" denote two elongated groups of $\mathrm{CH}_{3} \mathrm{OH}$ maser features. The dotted line outline the projection of the bipolar $\mathrm{H}_{2} \mathrm{O}$ maser jet onto the plane of the sky, while the dashed line is a linear fit to the $\mathrm{CH}_{3} \mathrm{OH}$ maser features of group 1 .

and trace more quiescient gas than the water masers. In the following we describe the results obtained towards the sources IRAS 20126+4104 and AFGL5142. In the former, the $6.7 \mathrm{GHz} \mathrm{CH}_{3} \mathrm{OH}$ masers might originate a the Keplerian disk, partly rotating with the disk material and partly being entrained into a slow disk-wind. In the latter, we found evidence that the $\mathrm{CH}_{3} \mathrm{OH}$ masers emerge from an infalling envelope at a radius of $\approx 300$ AU from the protostar.

\subsection{IRAS $20126+4104$}

Figure 4 shows positions and velocities of the $22 \mathrm{GHz} \mathrm{H}_{2} \mathrm{O}$ and $6.7 \mathrm{GHz} \mathrm{CH}_{3} \mathrm{OH}$ masers towards the intermediate-massive protostar IRAS 20126+4104. The $\mathrm{H}_{2} \mathrm{O}$ maser velocities trace a bipolar, collimated, NE-SW oriented outflow at a distance of a few hundred AU from the star. Moscadelli et al.(2011), by fitting a simple model of a conical jet, derive the main parameters of the water maser outflow: an outflow axis PA of $115^{\circ}$, an inclination with respect to the line-of-sight (LOS) of $80^{\circ}$, an opening angle of $18^{\circ}$, and the position of the central star (i.e., the cone vertex) with an accuracy of $\approx 100$ mas.

The $6.7 \mathrm{GHz}$ methanol masers are clustered to the NE of the star and their positions 
and LOS velocities are compatible with their origin from the northeastern side of the Keplerian disk detected in several high-density thermal tracers about the star (Cesaroni et al. 1997). Looking at Fig. 4, one sees that the $\mathrm{CH}_{3} \mathrm{OH}$ maser features are roughly outlining two linear structures, one oriented NE-SW (group 1) and the other SE-NW (group 2). Moscadelli et al. (2011) have calculated the relative proper motions (see Fig. 4) of the $\mathrm{CH}_{3} \mathrm{OH}$ maser features of both groups with respect to a feature belonging to group 1, which appears to be the most reliable for the persistency of the internal structure over the observing epochs.

It is worth noting two facts. First, the masers in group 2 move towards NW, i.e. in the same direction as the blue lobe of the $\mathrm{H}_{2} \mathrm{O}$ maser jet. Despite the different speeds (up to $\sim 100 \mathrm{~km} \mathrm{~s}^{-1}$ for $\mathrm{H}_{2} \mathrm{O}$ and only $\sim 5 \mathrm{~km} \mathrm{~s}^{-1}$ for $\mathrm{CH}_{3} \mathrm{OH}$ ), this suggests a relationship between the two. Second, the mean velocity of group 1 features along the line of sight is $-6.9 \mathrm{~km} \mathrm{~s}^{-1}$, which differs by $-3.4 \mathrm{~km} \mathrm{~s}^{-1}$ with respect to the systemic LSR velocity of $3.5 \mathrm{~km} \mathrm{~s}^{-1}$. The mean distance of the same features from the nominal position of the star (starred symbol in Fig. 4) is 0.27 or $\sim 460$ AU. If the masers are undergoing Keplerian rotation about the $7 M_{\odot}$ star, the expected velocity is $\sim-3.7 \mathrm{~km} \mathrm{~s}^{-1}$, consistent with the value of $-3.4 \mathrm{~km} \mathrm{~s}^{-1}$ quoted above.

Based on this, Moscadelli et al. (2011) proposed that group 1 masers trace the Keplerian disk, while for group 2 masers it is suggested that they also lie in the disk, but very close to the transition region between the disk surface and the outer part of the jet. This hypothesis may explain why the relative proper motions of group 2 features (see Fig. 4) are roughly perpendicular to the plane of the disk. Group 2 features could be tracing material lifted from the disk surface and accelerated along the rotation axis. This scenario is reminiscent of the MHD "disk wind", driven by the toroidal magnetic pressure (see, e.g., Banerejee \& Pudritz 2005 and references therein), invoked to explain the formation of large scale outflows in Young Stellar Objects.

\subsection{AFGL5142}

Figure 5 shows the proper motions of the $22 \mathrm{GHz}$ water masers (left panel) and $6.7 \mathrm{GHz}$ methanol masers (right panel) measured towards the millimeter core AFGL 5142 MM1 by Goddi et al. (2011). The high-angular resolution maps show a collimated bipolar outflow, at radii 140 to $400 \mathrm{AU}$, traced by radio continuum emission from its ionized component and by water masers from its molecular component. The two peaks of radio continuum emission are associated with the two clusters of water masers which are expanding, suggesting that the radio continuum probably comes from an ionized jet.

Looking at the right panel of Figure 5 , the $6.7 \mathrm{GHz} \mathrm{CH}_{3} \mathrm{OH}$ yellow spots have relatively large proper motions, directed towards the geometric center of the red features, which move mostly along the LOS, indicating motions towards the protostar. Since all methanol masers are seen in the foreground of the $22 \mathrm{GHz}$ continuum emission (which is optically thick at $6.7 \mathrm{GHz}$ ) and have red-shifted LOS velocities, they most likely trace infall toward the protostar. Goddi et al. (2011) have reproduced the methanol maser velocity distribution with a simple model of spherical infall, deriving a central mass of $4 \pm 1 \mathrm{M}_{\odot}$, a radius for the infalling envelope of $290 \pm 70 \mathrm{AU}$, an infall velocity of $5 \pm 1 \mathrm{~km} \mathrm{~s}^{-1}$, and a stellar position (i.e., the sphere center) coincident (within the fit error of 20 mas) with the geometric center of the red features. The derived mass infall rate of $6 \times 10^{-4} \mathrm{M}_{\odot} \mathrm{yr}^{-1}$ implies a protostellar age $\lesssim 10^{-4}$ yr. Note that methanol masers, for the first time, show evidence of infall of material on to the protostar from the $3-\mathrm{D}$ velocity field of molecular gas. 

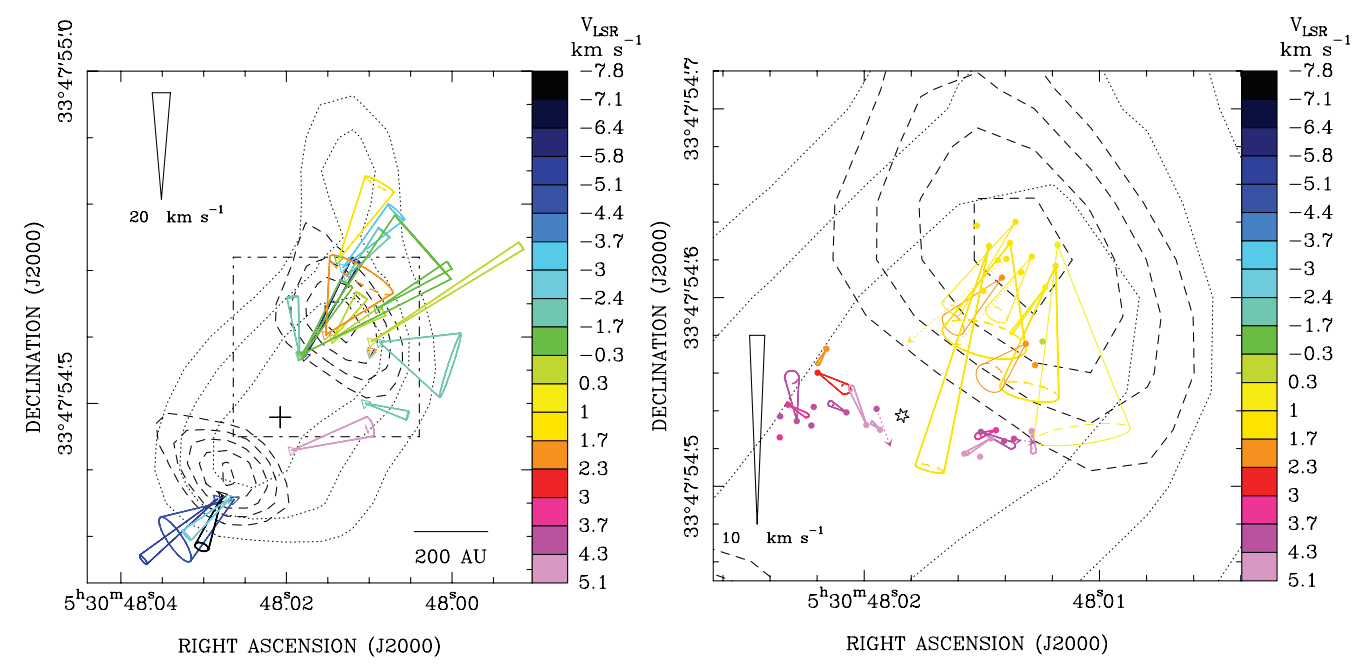

Figure 5. Proper motions of $\mathrm{H}_{2} \mathrm{O}$ (Left panel) and $\mathrm{CH}_{3} \mathrm{OH}$ (Right panel) masers measured towards AFGL 5142 MM-1. The dot-dashed rectangle in the left panel shows the area plotted in the right panel. The colored cones indicate proper motion orientation and uncertainties for individual maser features (the amplitude scale is given at the left of each panel). Colors denote line-of-sight velocities and contour maps show the $22 \mathrm{GHz}$ (dotted line) and $8.4 \mathrm{GHz}$ (dashed line) continuum emissions observed with the VLA. The black star in the right panel identifies the expected location of the protostar, corresponding to the geometric mean of positions of the red methanol masers. Water masers and radio continuum together identify a collimated bipolar (ionized/molecular) outflow.

\section{Summary}

1. Class II $\mathrm{CH}_{3} \mathrm{OH}$ masers are closely associated with massive star formation and are very good targets for astrometry because they are compact, long lived and slow moving. 2. Parallax accuracies of better than $10 \mu \mathrm{ms}$ have been obtained for $\mathrm{CH}_{3} \mathrm{OH}$ masers, indicating that they can be used to construct the spiral structure throughout the Milky Way's disk.

3. They can be used to study the Solar Motion and the Galaxy's rotation curve.

4. They can also be used to study the gas kinematics close to the exciting star, which might trace rotation, infall and/or outflowing motion.

\section{Acknowledgements}

This work was supported by the Chinese NSF through grants NSF 11133008, NSF 11073054, NSF 10621303, NSF 10733030 and the Key Laboratory for Radio Astronomy, CAS. K.L.J.R is funded by an ASI fellowship under contract number I/005/07/1.

\section{References}

Banerjee, R. \& Pudritz, R. E. 2005, "Outflows and Jets from Collapsing Magnetized Cloud Cores", Protostars and Planets V, 2005.

Bartkiewicz, A., Brunthaler, A., Szymczak, M. van Langevelde, H. J \& Reid, M. J. 2008, A\&্A, 490, 787

Brunthaler, A., Reid, M. J, Menten, K. M. et al. 2011, AN, 332, 461

Brunthaler, A., Reid, M. J., Menten, K. M., Zheng, X. W., Moscadelli, L., \& Xu, Y. 2009, ApJ, 693,424

Cesaroni, R., Felli, M., Testi, L., Walmsley, C. M., \& Olmi, L. 1997, A\&̈A, 325, 725 
Dehnen, W. \& Binney, J. J., MNRAS, 1998, 298,387

Georgelin, Y. M. \& Georgelin, Y. P., 1976, A\& $A, 49,57$

Goddi, C., Moscadelli, L., \& Sanna, A. 2011, A\&A, 535, L8.

Moscadelli, L., Reid, M. J., Menten, K. M., Brunthaler, A., Zheng, X. W., \& Xu, Y. 2009, ApJ, 693,406

Moscadelli, L., Cesaroni, R., Rioja, M. J., Dodson, R., \& Reid, M. J. 2011, A\&A, 526, 66

Nagayama, T., Omodaka, T., Nakagawa, A., et al. 2011, PASJ, 63, 23

Reid, M. J., Menten, K. M., Brunthaler, A., Zheng, X. W., Moscadelli, L., \& Xu, Y. 2009, ApJ, 693,397

Reid, M. J., Menten, K. M., Zheng, X. W. et al. 2009, ApJ, 700, 137

Rygl, K. L. J., Brunthaler, A., Reid, M. J., Menten, K. M., van Langevelde, H. J., \& Xu, Y. 2010, $A \& A, 511,2$

Rygl, K. L. J., Brunthaler, A., Sanna, A. et al. 2012, A\&A, 539, 79

Sanna, A., Reid, M. J., Moscadelli, L., Dame, T. M., Menten, K. M., Brunthaler, A., Zheng, X. W., \& Xu, Y. 2009, ApJ, 706, 464

Sanna, A., Moscadelli, L., Cesaroni, R., Tarchi, A., Furuya, R. S., \& Goddi, C. 2010, A\&A, 517, A78

Sato, M., Hirota, T., Honma, M., et al. 2008, PASJ, 60, 975

Schönrich, R., Binney, J. J., \& Dehnen, W., 2010, MNRAS, 403, 1829

Xu, Y., Reid, M. J., Zheng, W. W., \& Menten, K. M. 2006, Science, 311, 54

Xu, Y., Reid, M. J., Menten, K. M., Brunthaler, A., Zheng, X. W., \& Moscadelli, L. 2009, ApJ, 693,413

Xu, Y., Moscadelli, L., Reid, M. J. et al., 2011, ApJ, 733, 25

Zhang, B., Zheng, X. W., Reid, M. J., Menten, K. M., Xu, Y., Moscadelli, L., \& Brunthaler, A. 2009, ApJ, 693, 419 